\title{
Passive Immunoprophylaxis with Specific Monoclonal Antibody Confers Partial Protection against Pneumocystis carinii Pneumonitis in Animal Models
}

\author{
Francis Gigliotti and Walter T. Hughes
}

Department of Infectious Diseases, St. Jude Children's Research Hospital, Memphis, Tennessee 38101

\begin{abstract}
Pneumocystis carinii is an important cause of pneumonitis in the immunosuppressed host. Little is known, however, about the biology of this organism. This report demonstrates that a MAb, M5E12, previously shown to be directed against a surface antigen that is present on rat-, rabbit-, ferret-, and human-derived $\boldsymbol{P}$. carinii, is capable of hindering the development of $\boldsymbol{P}$. carinii pneumonitis in animal models of this infection when administered throughout the period of immunosuppression. It appears that MAb M5E12 thus has identified a surface antigen of $\boldsymbol{P}$. carinii that is important in host-parasite interactions.
\end{abstract}

\section{Introduction}

In spite of the fact that Pneumocystis carinii is a well-recognized cause of pneumonia in immunosuppressed patients, relatively little is known about the biology of this organism or about the immunopathogenesis of $P$. carinii pneumonitis (PCP). ${ }^{1}$ Information about $P$. carinii has been greatly limited because of the inability to establish long-term or cell-free cultures of the organism and by the lack of specific, well-characterized serologic reagents (1). Observations made from animal models have led to the assumption that cell-mediated immunity is crucial in protection from PCP, whereas humoral immunity is of little or no significance (2-4). A role for humoral immunity, however, is suggested by the finding that natural infection with $P$. carinii occurs in humans with pure humoral as well as cellular immune defects (5), and that experimental animals develop both systemic and local antibody responses during recovery from PCP (6). In addition, passive administration of rabbit serum obtained after immunization with $P$. carinii delayed death from PCP in one study using nude mice (2).

Highly specific murine MAbs to $P$. carinii can be produced without the need for culturing or purifying these organisms (7). Characterization of the binding specificity of these and other MAbs has established that they generally react with $P$. carinii that is derived only from the animal species from which the immunizing organisms were obtained $(7,8)$. Some MAbs, however, do react with organisms obtained from different spe-

Address reprint requests to Dr. Francis Gigliotti, St. Jude Children's Research Hospital, Department of Infectious Diseases, 332 N. Lauderdale Street, Memphis, TN 38101.

Received for publication 26 September 1987 and in revised form 16 December 1987.

1. Abbreviations used in this paper: $\mathrm{PCP}$, Pneumocystis carinii pneumonitis.

J. Clin. Invest.

(c) The American Society for Clinical Investigation, Inc.

$0021-9738 / 88 / 06 / 1666 / 03 \quad \$ 2.00$

Volume 81, June 1988, 1666-1668 cies $(7,8)$. MAb M5E12 has been shown to bind to $P$. carinii of rat, rabbit, ferret, and human origin (7). The surface antigen of ferret-derived $P$. carinii recognized by this IgM MAb has been partially characterized and is a glycoprotein, containing disulfide linkages, which is bound by Con $A$ and wheat germ agglutinin (unpublished observations). Because the epitope recognized by MAb M5E12 appears to be conserved on $P$. carinii obtained from diverse hosts, it was a likely monoclonal for testing the effect of passive antibody immunoprophylaxis in the ferret and rat models of $\operatorname{PCP}(7,9)$. In both of these models, the animals develop spontaneous PCP upon immunosuppression. Within 2 wk PCP is demonstrable in the ferrets (unpublished observations); rats require at least 6-8 wk of immunosuppression before PCP is evident.

\section{Methods}

Passive immunoprophylaxis of ferret PCP. For these experiments, young ferrets weighing $\sim 0.5-0.9 \mathrm{~kg}$ (Marshall Research Animals, Inc., North Rose, NY) were immunosuppressed by placing $2 \mathrm{mg} /$ liter dexamethasone (Elkins-Sinn, Inc., Cherry Hill, NJ) in their drinking water as described for the rat model (7). The animals were maintained on steroids throughout the duration of these experiments. MAb M5E12 and control IgM MAb 5M1H9, directed against the capsular polysaccharide of $H$. influenzae type b (10), were prepared by saturated ammonium sulfate precipitation of ascites fluid followed by extensive dialysis against PBS. MAb 5M1H9 was chosen because it is of the same isotype as MAb M5E12 and it does not bind to $P$. carinii, as determined by immunofluorescence. The amount of IgM protein in each preparation was approximated by an RIA and the MAb preparations were also titered against $P$. carinii by indirect immunofluorescence assay (7). At the end of each experiment, the animals were killed and their lungs removed. To count the number of $P$. carinii cysts, $1 \mathrm{~g}$ of lung was carefully homogenized, stained with toluidine blue $O$, coded, and then the cysts were counted as described (7).

Two experiments were performed in ferrets. In the first set of experiments, steroid-induced, immunosuppressed ferrets were given weekly intravenous injections of either MAb M5E12 or $5 \mathrm{M} 1 \mathrm{H} 9$, at a dosage of $\sim 300 \mu \mathrm{g} \mathrm{IgM/kg}$. This arbitrary dosage was based on the volume of MAb M5E12 that could be easily administered intravenously. Antibody administration was begun during the first week of immunosuppression. $1 \mathrm{wk}$ after the eighth injection of antibody, the animals were killed and the number of $P$. carinii cysts in their lungs was counted. In the second experiment, animals were injected three times per week with MAb M5E12 at a dosage of $\sim 200 \mu \mathrm{g} \mathrm{IgM/kg}$. Control animals again received antibody $5 \mathrm{M} 1 \mathrm{H} 9$, but this time the dosage of IgM was $\sim 10$-fold higher than that received by the experimental group. Treatments were continued for $3 \mathrm{wk}$ (nine injections). 3 d after the last injection, the animals were killed and the extent of PCP was quantitated.

Passive immunoprophylaxis of murine PCP. Sprague-Dawley rats (Harlan Sprague Dawley, Inc., Indianapolis, IN), weighing 200-300 g, were used to determine the effect of passively administered MAb M5E12 on this widely used animal model of PCP. The rats, immunosuppressed identically to the ferrets, received $200 \mu \mathrm{g} \mathrm{IgM} / \mathrm{kg}$ of M5E 12 i.p. once weekly for $3 \mathrm{wk}$. Starting with the fourth week of immunosuppression, the dosage of antibody was increased to $300 \mu \mathrm{g} \mathrm{IgM/kg} \mathrm{ad-}$ ministered thrice weekly for $9 \mathrm{wk}$. Control animals for this experiment received $0.5 \mathrm{ml} / \mathrm{kg}$ once per week of an irrelevant $\mathrm{IgG} \mathrm{MAb}$ (11) 
prepared from ascites fluid. After the twelfth week of immunosuppression rats were killed and the total number of $P$. carinii cysts in the lungs of each animal was quantitated as noted above.

Western blot analysis. Organisms obtained from ferrets that received either MAb M5E12 or control MAb 5M1H9 were examined for the presence of the surface antigen recognized by MAb M5E12 by Western blot. The specificity of this Western blot for a $P$. carinii antigen has been previously described (7). The only modification of the Western blot used in this study was that the organisms were solubilized by boiling for $15 \mathrm{~min}$ in the cracking buffer rather than by sonication.

\section{Results}

As can be seen in Table I, passive immunoprophylaxis of ferrets with MAb M5E12 resulted in a marked reduction in the number of $P$. carinii organisms present in their lungs. In the first experiment, in which antibody was administered once weekly over a period of 2 mo, ferrets receiving MAb M5E12 had a 20 -fold reduction in the number of $P$. carinii per gram of lung tissue ( $P=0.02$; Mann-Whitney rank sum test). Likewise, animals receiving MAb M5E12 twice weekly, but for a total duration of only $3 \mathrm{wk}$, had a 10 -fold reduction in the number of $P$. carinii when compared with control animals receiving MAb 5M1H9 ( $P=0.13$; Mann-Whitney rank sum test). When analyzed together, the two ferret experiments show that administration of MAb M5E12 resulted in a $90 \%$ reduction in the geometric mean number of organisms present in $1 \mathrm{~g}$ of lung tissue from $4.1 \times 10^{6}$ organisms $/ g$ in the control group to $3.4 \times 10^{5}$ organisms $/ g$ in the experimental group $(P=0.01$; Mann-Whitney rank sum test).

A parallel experiment was performed to examine the ex-

Table I. Effect of MAb MSE12 on the Development of P. carinii Pneumonitis in Immunosuppressed Ferrets

\begin{tabular}{|c|c|c|c|}
\hline \multicolumn{2}{|c|}{ M5E12 Group } & \multicolumn{2}{|c|}{ 5M1H9 Group } \\
\hline Animal No. & $\begin{array}{l}\text { P. cariniil } \\
\text { gram of lung }\end{array}$ & Animal No. & $\begin{array}{l}\text { P. carinii/ } \\
\text { gram of lung }\end{array}$ \\
\hline \multicolumn{4}{|l|}{ Exp. 1} \\
\hline 549 & $7.5 \times 10^{5}$ & 554 & $9.4 \times 10^{5}$ \\
\hline 551 & $4.3 \times 10^{4}$ & 556 & $1.3 \times 10^{7}$ \\
\hline 553 & $1.3 \times 10^{5}$ & 566 & $5.8 \times 10^{6}$ \\
\hline 565 & $1.6 \times 10^{5}$ & 567 & $1.4 \times 10^{6}$ \\
\hline Geometric mean* & $1.6 \times 10^{5}$ & & $3.2 \times 10^{6}$ \\
\hline \multicolumn{4}{|l|}{ Exp. 2} \\
\hline 661 & $3.7 \times 10^{7}$ & 667 & $1.1 \times 10^{6}$ \\
\hline 662 & $2.8 \times 10^{5}$ & 668 & $3.0 \times 10^{5}$ \\
\hline 663 & $2.7 \times 10^{5}$ & 671 & $1.0 \times 10^{7}$ \\
\hline 664 & $7.4 \times 10^{3}$ & 672 & $9.7 \times 10^{6}$ \\
\hline 665 & $7.4 \times 10^{4}$ & 673 & $3.3 \times 10^{7}$ \\
\hline 666 & $1.1 \times 10^{7}$ & 674 & $1.5 \times 10^{7}$ \\
\hline 669 & $2.7 \times 10^{4}$ & 675 & $3.5 \times 10^{6}$ \\
\hline 670 & $6.9 \times 10^{6}$ & - & - \\
\hline Geometric mean* & $4.9 \times 10^{5}$ & & $5.6 \times 10^{6}$ \\
\hline $\begin{array}{l}\text { Cumulative geomet } \\
\text { mean* }\end{array}$ & $3.4 \times 10^{5}$ & & $4.1 \times 10^{6}$ \\
\hline
\end{tabular}

* The statistical difference between the geometric means of the two groups as determined by the Mann-Whitney rank sum test are, Exp. $1, P=0.02$; Exp. 2, $P=0.13$; and Cumulative, $P=0.01$.
Table II. Effect of MAb M5E12 on the Development of P. carinii Pneumonitis in Immunosuppressed Rats

\begin{tabular}{|c|c|c|c|}
\hline \multicolumn{2}{|c|}{ M5E12 Group } & \multicolumn{2}{|c|}{ M2E2 Group } \\
\hline Animal No.* & $\begin{array}{l}\text { P. carinii/ } \\
\text { whole lungs }\end{array}$ & Animal No. & $\begin{array}{c}\text { P. carinii/ } \\
\text { whole lungs }\end{array}$ \\
\hline 1 & $3.6 \times 10^{6}$ & 1 & $1.2 \times 10^{7}$ \\
\hline 2 & $7.2 \times 10^{6}$ & 2 & $3.5 \times 10^{7}$ \\
\hline 3 & $1.3 \times 10^{7}$ & 3 & $1.4 \times 10^{7}$ \\
\hline 4 & $7.9 \times 10^{6}$ & 4 & $3.3 \times 10^{7}$ \\
\hline 5 & $1.6 \times 10^{6}$ & 5 & $3.2 \times 10^{7}$ \\
\hline 6 & $3.9 \times 10^{6}$ & 6 & $2.7 \times 10^{7}$ \\
\hline 7 & $1.7 \times 10^{6}$ & & \\
\hline 8 & $8.3 \times 10^{6}$ & & \\
\hline 9 & $5.6 \times 10^{6}$ & & \\
\hline Geometric mean ${ }^{\ddagger}$ & $4.8 \times 10^{6}$ & & $2.4 \times 10^{7}$ \\
\hline
\end{tabular}

* One animal in M5E12 group and four animals in the M2E2 group died before the last week of the experiment.

\# The statistical difference between the geometric means of the two groups as determined by the Mann-Whitney rank sum test is $P$ $=0.002$.

tent of PCP in untreated immunosuppressed ferrets as a function of the duration of immunosuppression. Seven ferrets killed between 3 and $10 \mathrm{wk}$ after initiation of steroid treatment all had $\geq 10^{6}-10^{7} P$. carinii cysts/g of lung tissue. 16 of 18 animals that received either no antibody or control antibody thus had $\geq 10^{6} P$. carinii cysts/g of lung compared with only 3 of 12 that had received MAb M5E12 $(P<0.001$ by Fisher's exact test).

There was a similar reduction in the number of organisms present in the lungs of rats receiving passive immunoprophylaxis with MAb M5E12 (Table II). Rats receiving MAb M5E12 had an approximately fivefold reduction in the number of organisms when compared with control animals $(P=0.002$ by Mann-Whitney rank sum test).

Three ferrets (Table I, animals No. 661, 666, and 670) clearly received no benefit from MAb M5E12. The reason for this is unclear, but may have important biologic implications. To exclude the possibility of antigenic modulation or variation, which is common among protozoan parasites, (12) Western blot analysis was performed on $P$. carinii obtained from ferrets in each group. Fig. 1 clearly demonstrates that when an equal number of $P$. carinii is solubilized, the $115-120-\mathrm{kD}$ glycoprotein recognized by MAb M5E12 is present, regardless of which antibody the ferret received.

\section{Discussion}

While of a preliminary nature, these experiments demonstrate that, contrary to generally held beliefs, enhancement of humoral immunity can significantly alter the course of $P$. carinii infection. In both the ferret and rat models of PCP, passive administration of relatively small amounts of $P$. carinii-specific IgM MAb resulted in a lessening of the intensity of infection with $P$. carinii. MAb M5E12 appeared to have a more pronounced effect in the ferret model. One possible explanation for the apparently reduced effect of MAb M5E12 in the rat model is a lesser affinity of the antibody for rat versus ferret $P$. carinii. Alternatively, there could be a differential expres- 


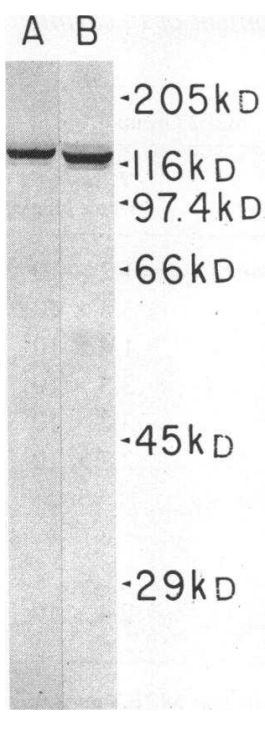

Figure 1. Western blot analysis of equal numbers of $P$. carinii obtained from a ferret (No. 666) that received MAb M5E12, but still developed extensive PCP (lane $A$ ), and from a control ferret (No. 674) that received MAb 5M1H9 (lane $B$ ). The antigen recognized by MAb M5E12 is visualized in both lanes.

sion of the antigen recognized by MAb M5E12 on organisms obtained from these two species. When titered by immunofluorescence assay MAb M5E12 has an $\sim 100$-fold higher titer when ferret $P$. carinii are used as the target instead of rat $P$. carinii. Likewise, when analyzed by Western blot, MAb M5E12 produces a much stronger blot with ferret-derived $P$. carinii compared with an equal number of rat-derived $P$. carinii (7). Finally, the pathology in the ferret model of PCP is an interstitial pneumonitis without the foamy alveolar exudates typically seen in rat PCP (9). Whether these differences contributed to the more pronounced effect in the ferret model is not known.

There are several possible explanations for why the administration of MAb M5E12 did not completely prevent the development of PCP in this system. First, the animals are maintained on immunosuppressive therapy throughout the experiment, so that administration of relatively small amounts of an MAb to a single epitope on $P$. carinii may not allow for sufficient immunoreconstitution to prevent this infection altogether. Secondly, while MAb M5E12 clearly recognizes cyst forms of $P$. carinii (7), immunofluorescent assays on $P$. carinii-infected lung lavage that has been passed through 3.0- and $1.2-\mu \mathrm{m}$ filters demonstrate staining of structures consistent in size with trophozoites (unpublished observation). It is possible that MAb in 5E12 may exert its protective effect, e.g., complement-mediated cell lysis or inhibition of attachment, on only the trophozoite stage (13). Any trophozoite that escapes attack by MAb M5E12 thus will go on to produce a mature cyst which will then give rise to eight additional organisms. As demonstrated by Fig. 1, antigenic modulation does not explain the failure to totally protect against PCP. Further studies will be necessary to determine the mechanism of protection afforded by MAb M5E12. Whether the results described here can be improved by alteration in dosages of antibody, adding MAbs directed against other surface antigens, or by combination immunotherapy using other biologic response modifiers will need to be determined.

Besides indicating that humoral immunity to $P$. carinii merits further investigation, these studies also give the first specific information yet available about antigens on the surface of $P$. carinii. Several investigators have now reported MAbs or polyclonal antibodies that recognize a $P$. carinii antigen of $\sim 110,000-120,000 \mathrm{D}$ in size $(7,14-16)$. There are insufficient comparative data to determine whether these reports are describing the same antigen, but the frequent finding of antibody to a similar size antigen of $P$. carinii suggests that this may be an important surface molecule. Use of MAbs such as M5E12 thus will aid in identifying, characterizing, and purifying antigens on $P$. carinii, even in the absence of adequate culture systems, which are clearly important in host-parasite interactions.

\section{Acknowledgments}

We wish to acknowledge the expert technical assistance of Bernetta Moseley and thank Dr. Alan Brown for performing the IgM quantitations and Richard Dodge for statistical analysis.

This work was supported by grant AI-23302 from the National Institute of Allergy and Infectious Diseases and by the American Lebanese Syrian Associated Charities.

\section{References}

1. Young, L. S. 1984. Pneumocystis carinii Pneumonia. Marcel Dekker, Inc., New York.

2. Furuta, T., K. Ueda, S. Kyuwa, and K. Fujiwara. 1984. Effect of T-cell transfer on Pneumocystis carinii infection in nude mice. Jpn. J. Exp. Med. 54:57-64.

3. Furuta, T., K. Ueda, and K. Fujiwara. 1984. Experimental Pneumocystis carinii infection in nude rats. Jpn. J. Exp. Med. 54:6572.

4. Furuta, T., K. Ueda, K. Fujiwara, and K. Yamanouchi. 1985. Cellular and humoral immune responses of mice subclinically infected with Pneumocystis carinii. Infect. Immun. 47:544-548.

5. Burke, B. A., and R. A. Good. 1973. Pneumocystis carinii infection. Medicine (Baltimore): 52:23-51.

6. Walzer, P. D., and M. E. Rutledge. 1981. Humoral immunity in experimental Pneumocystis carinii infection. J. Lab. Clin. Med. 97:820-833.

7. Gigliotti, F., D. C. Stokes, A. B. Gheatham, D. S. Davis, and W. T. Hughes. 1986. Development of murine monoclonal antibodies to $P$. carinii. J. Infect. Dis. 154:315-322.

8. Kovacs, J. A., J. C. Swan, V. Gill, J. E. Parrillo, and H. Masur. 1986. Monoclonal antibodies against Pneumocystis carinii: utility for establishing diagnosis and species differences. 26th Intersci. Conf. Antimicrobial Agents Chemother. (Abstr.)

9. Stokes, D. C., F. Gigliotti, J. F. Rehg, R. L. Snellgrove, and W. T. Hughes. 1987. Experimental Pneumocystis carinii pneumonia in the ferret. Br. J. Exp. Pathol. 68:267-276.

10. Gigliotti, F., and R. A. Insel. 1982. Protection from infection with Haemophilus influenzae type b by monoclonal antibody to the capsule. J. Infect. Dis. 146:249-254.

11. Gigliotti, F., and J. L. Shenep. 1985. Failure of monoclonal antibodies to core glycolipid to bind intact smooth strains of Escherichia coli. J. Infect. Dis. 151:1005-1011.

12. Kagan, I. G., and S. E. Maddison. 1982. Immunology of human infection. Part II: Viruses and parasites; immunodiagnosis and prevention of infectious diseases. In Comprehensive Immunology. A. J. Nahmias and R. J. O'Reilly, editors. Plenum Publishing Corp., New York. 1315-1325.

13. Hughes, W. T. 1984. Pneumocystis carinii: Biology and mode of transmission. In Acquired Immunodeficiency Syndrome. M. S. Gottlieb and J. E. Groopman, editors. Alan R. Liss, Inc., New York. 345-354.

14. Lee, C. H., C. D. Bolinger, M. S. Bartlett, R. B. Kohler, C. E. Wilde, and J. W. Smith. 1986. Production of monoclonal antibody against Pneumocystis carinii by using a hybrid of rat spleen and mouse myeloma cells. J. Clin. Microbiol. 23:505-508.

15. Graves, D. C., S. J. N. McNabb, M. H. Ivey, and M. A. Worley. 1986. Development and characterization of monoclonal antibodies to Pneumocystis carinii. Infect. Immun. 51:125-133.

16. Walzer, P. D., and M. J. Linke. 1987. A comparison of the antigenic characteristics of rat and human Pneumocystis carinii by immunoblotting. J. Immunol. 138:2257-2265. 